NSF-ITP-94-44

\title{
Catalysis of Dynamical Flavor Symmetry Breaking by a Magnetic Field in $2+1$ Dimensions
}

\author{
V.P. Gusynin ${ }^{1}$, V.A. Miransky ${ }^{1,2}$, and I.A. Shovkovy ${ }^{1}$ \\ ${ }^{1}$ Bogolyubov Institute for Theoretical Physics, 252143 Kiev, Ukraine \\ ${ }^{2}$ Institute for Theoretical Physics, University of California, \\ Santa Barbara, CA 93106-4030
}

\begin{abstract}
It is shown that in $2+1$ dimensions, a constant magnetic field is a strong catalyst of dynamical flavor symmetry breaking, leading to generating a fermion dynamical mass even at the weakest attractive interaction between fermions. The effect is illustrated in the Nambu-Jona-Lasinio model in a magnetic field. The low-energy effective action in this model is derived and the thermodynamic properties of the model are established. The relevance of this effect for planar condensed matter systems is pointed out.
\end{abstract}


Relativistic field models in $2+1$ dimensional space-time have been a subject of considerable interest: Their sophisticated dynamics is interesting in itself; they also serve as effective theories for the description of long wavelength excitations in planar condensed matter systems $[1,2]$; also, their dynamics imitates the dynamics of $3+1$ dimensional theories at high temperature.

In this Letter, we will show that a constant magnetic field acts as a strong catalyst of dynamical flavor symmetry breaking in $2+1$ dimensions, leading to generating a fermion dynamical mass even at the weakest attractive interaction between fermions. We would like to stress that this effect is universal, i.e., model independent, in $2+1$ dimensions. This point may be important in connection with considering this effect in such condensed matter phenomena as the quantum Hall effect [1] and high temperature superconductivity [2], and in $3+1$ dimensional theories at high temperature.

Let us begin by considering the basic points in the problem of a relativistic fermion in a constant magnetic field $B$ in $(2+1)$-dimensions. The Lagrangian density is

$$
\mathcal{L}=\frac{1}{2}\left[\bar{\Psi},\left(i \gamma^{\mu} D_{\mu}-m\right) \Psi\right]
$$

where the covariant derivative $D_{\mu}$ is

$$
D_{\mu}=\partial_{\mu}-i e A_{\mu}^{e x t}, \quad A_{\mu}^{e x t}=-B x_{2} \delta_{\mu 1}
$$

We use four-component spinors corresponding to a reducible representation of the Dirac algebra [3]:

$$
\gamma^{0}=\left(\begin{array}{cc}
\sigma_{3} & 0 \\
0 & -\sigma_{3}
\end{array}\right), \gamma^{1}=\left(\begin{array}{cc}
i \sigma_{1} & 0 \\
0 & -i \sigma_{1}
\end{array}\right), \gamma^{2}=\left(\begin{array}{cc}
i \sigma_{2} & 0 \\
0 & -i \sigma_{2}
\end{array}\right)
$$

At $m=0$, the Lagrangian (1) is invariant under the flavor $U(2)$ transformations with generators $T_{0}=I, T_{1}=\gamma^{5}, T_{2}=\frac{1}{i} \gamma^{3}, T_{3}=\gamma^{3} \gamma^{5}$ where $\gamma^{5}=i \gamma^{0} \gamma^{1} \gamma^{2} \gamma^{3}$. The mass term breaks $U(2)$ down to $U(1) \times U(1)$ with generators $T_{0}$ and $T_{3}$. 
Our basic observation is that in $2+1$ dimensions, as $m \rightarrow 0$, spontaneous $U(2)$ symmetry breaking takes place for fermions in a constant magnetic field. In order to prove this, we will show that in the limit $m \rightarrow 0$, the flavor condensate $\langle 0|\bar{\Psi} \Psi| 0\rangle$ is nonzero: $\langle 0|\bar{\Psi} \Psi| 0\rangle=-\frac{|e B|}{2 \pi}$.

The condensate is expressed through the fermion propagator $S(x, y)=\langle 0|T(\Psi(x) \bar{\Psi}(y))| 0\rangle$. The propagator $S$ can be calculated by using the Schwinger (proper time) approach [4]. It is

$$
S(x, y)=\exp \left(i e \int_{y}^{x} A_{\lambda}^{e x t} d z^{\lambda}\right) \tilde{S}(x-y)
$$

where the integral is calculated along the straight line, and the Fourier transform of $\tilde{S}(x)$ (in Euclidean space) is

$$
\begin{aligned}
\tilde{S}(k) & =-i \int_{0}^{\infty} d s \exp \left[-s\left(m^{2}+k_{3}^{2}+\mathbf{k}^{2} \frac{\tanh (e B s)}{e B s}\right)\right]\left(-k_{\mu} \gamma_{\mu}+m+\right. \\
& \left.+\frac{1}{i}\left(k_{2} \gamma_{1}-k_{1} \gamma_{2}\right) \tanh (e B s)\right)\left(1+\frac{1}{i} \gamma_{1} \gamma_{2} \tanh (e B s)\right)
\end{aligned}
$$

$\left(k_{3}=-i k^{0}\right.$ and $\gamma_{\mu}$ are antihermitian matrices). The condensate is

$$
\begin{aligned}
\langle 0|\bar{\Psi} \Psi| 0\rangle & =-\lim _{x \rightarrow y} \operatorname{tr} S(x, y)=-\frac{i}{(2 \pi)^{3}} \operatorname{tr} \int d^{3} k \tilde{S}(k)=-\lim _{\Lambda \rightarrow \infty} \frac{4 m}{(2 \pi)^{3}} \int d^{3} k \int_{1 / \Lambda^{2}}^{\infty} d s \\
& \cdot \exp \left[-s\left(m^{2}+k_{3}^{2}+\mathbf{k}^{2} \frac{\tanh (e B s)}{e B s}\right)\right] \overrightarrow{m \rightarrow 0}-\frac{|e B|}{2 \pi}
\end{aligned}
$$

( $\Lambda$ is an ultraviolet cutoff). Thus in a constant magnetic field, spontaneous breakdown of the $U(2)$ symmetry takes place even though fermions do not acquire a dynamical mass. We note that in $3+1$ dimensions, the result would be $\langle 0|\bar{\Psi} \Psi| 0\rangle \sim m \ln m \longrightarrow 0$ as $m \rightarrow 0$. Therefore, this is a specific $2+1$ dimensional phenomenon.

What is the physical basis of this phenomenon? In order to answer this question, we note that the singular, $\frac{1}{m}$, behavior of the integral in Eq. (6) is formed at large, $s \rightarrow \infty$, distances ( $s$ is the proper time coordinate). Actually, one can see from Eq. (6) that the magnetic field effectively removes the two space dimensions in the infrared region, thus reducing the 
dynamics to a one-dimensional dynamics which has much more severe infrared singularities. From this viewpoint, the action of the magnetic field in this problem is similar to that of the Fermi surface in the BCS theory [5].

This point is intimately connected with the form of the energy spectrum of fermions in a constant magnetic field. In $(2+1)$-dimensions, it is [6]:

$$
E_{n}= \pm \sqrt{m^{2}+2|e B| n}, \quad n=0,1, \ldots
$$

(the Landau levels). Each Landau level is degenerate. At the lowest level with $n=0$, the density of states is $\frac{|e B|}{2 \pi}$; at the levels with $n \geq 1$, it is $\frac{|e B|}{\pi}$. As $m \rightarrow 0$, the energy $E_{0}$ goes to zero and therefore there is the infinite vacuum degeneracy in this case.

By using the explicit solution for the field $\Psi$ in this problem [6], we find the charge operators connected with the broken symmetry:

$$
\begin{aligned}
Q_{1}= & \frac{1}{2} \int d^{2} x\left[\Psi^{\dagger}(x), T_{1} \Psi(x)\right]=i \sum_{p}\left(a_{0 p}^{\dagger} d_{0-p}^{\dagger}-d_{0-p} a_{0 p}\right) \\
& +i \sum_{n=1}^{\infty} \sum_{p}\left[\left(a_{n p}^{\dagger} c_{n p}-c_{n p}^{\dagger} a_{n p}\right)+\left(b_{n p}^{\dagger} d_{n p}-d_{n p}^{\dagger} b_{n p}\right)\right] \\
Q_{2}= & \frac{1}{2} \int d^{2} x\left[\Psi^{\dagger}(x), T_{2} \Psi(x)\right]=\sum_{p}\left(a_{0 p}^{\dagger} d_{0-p}^{\dagger}+d_{0-p} a_{0 p}\right) \\
& +\sum_{n=1}^{\infty} \sum_{p}\left[\left(a_{n p}^{\dagger} c_{n p}+c_{n p}^{\dagger} a_{n p}\right)+\left(b_{n p}^{\dagger} d_{n p}+d_{n p}^{\dagger} b_{n p}\right)\right] .
\end{aligned}
$$

Here $a_{n p}, c_{n p}\left(b_{n p}, d_{n p}\right)$ are annihilation operators of fermions (antifermions) from the $n$-th Landau level (there are two types of fermions (antifermions) for the four-component fielt $\Psi$ ); the momentum $k=2 \pi p / L_{1}(p=0, \pm 1, \ldots), L_{1}$ is the size in the $x_{1}$-direction. A set of the degenerate vacua $\left|\theta_{1}, \theta_{2}\right\rangle$ is $\left|\theta_{1}, \theta_{2}\right\rangle \equiv \exp \left(i Q_{1} \theta_{1}+i Q_{2} \theta_{2}\right)|0\rangle$ where $|0\rangle$ satisfies the condition $a_{n p}|0\rangle=b_{n p}|0\rangle=c_{n p}|0\rangle=d_{n p}|0\rangle=0$. As $L_{1} \rightarrow \infty$, the vacua with different $\left\{\theta_{1}, \theta_{2}\right\}$ become orthogonal and define (as usual in the case of spontaneous symmetry breaking) nonequivalent representations of canonical commutation relations.

In each Fock space defined by a vacuum $\left|\theta_{1}, \theta_{2}\right\rangle$, there are a lot of "excitations" with nonzero momentum and zero energy created by the operators $a_{0 p}^{\dagger}, d_{0 p}^{\dagger}$. However, there are no 
genuine (i.e. with a nontrivial dispersion relation) Nambu-Goldstone (NG) modes: At the lowest Landau level, the energy $E=0$ (since the Lorentz symmetry is broken by a magnetic field in this problem, there is no contradiction with Goldstone's theorem). We shall show below that even the weakest attractive interaction between fermions and antifermions is enough to "resurrect" these modes (and a dynamical mass for fermions).

Let us consider the $2+1$ dimensional Nambu-Jona-Lasinio (NJL) model with the $U(2)$ flavor symmetry:

$$
\mathcal{L}=\frac{1}{2}\left[\bar{\Psi},\left(i \gamma^{\mu} D_{\mu}\right) \Psi\right]+\frac{G}{2}\left[(\bar{\Psi} \Psi)^{2}+\left(\bar{\Psi} i \gamma^{5} \Psi\right)^{2}+\left(\bar{\Psi} \gamma^{3} \Psi\right)^{2}\right]
$$

where $D_{\mu}$ is the covariant derivative (叉) and fermion fields carry an additional, "color", index $\alpha=1,2, \ldots, N_{c}$. This theory is equivalent to a theory with the Lagrangian density

$$
\begin{aligned}
\mathcal{L} & =\frac{1}{2}\left[\bar{\Psi},\left(i \gamma^{\mu} D_{\mu}\right) \Psi\right]-\bar{\Psi}\left(\sigma+\gamma^{3} \tau+i \gamma^{5} \pi\right) \Psi \\
& -\frac{1}{2 G}\left(\sigma^{2}+\pi^{2}+\tau^{2}\right)
\end{aligned}
$$

$\left(\sigma=-G(\bar{\Psi} \Psi), \tau=-G\left(\bar{\Psi} \gamma^{3} \Psi\right), \pi=-G\left(\bar{\Psi} i \gamma^{5} \Psi\right)\right)$. The effective action for these composite fields is:

$$
\Gamma(\sigma, \tau, \pi)=-\frac{1}{2 G} \int d^{3} x\left(\sigma^{2}+\tau^{2}+\pi^{2}\right)+\tilde{\Gamma}(\sigma, \tau, \pi)
$$

where

$$
\tilde{\Gamma}(\sigma, \tau, \pi)=-i \operatorname{Tr} \operatorname{Ln}\left[i \gamma^{\mu} D_{\mu}-\left(\sigma+\gamma^{3} \tau+i \gamma^{5} \pi\right)\right] .
$$

As $N_{c} \rightarrow \infty$, the path integral over the composite fields is dominated by stationary points of their action: $\frac{\delta \Gamma}{\delta \sigma}=\frac{\delta \Gamma}{\delta \tau}=\frac{\delta \Gamma}{\delta \pi}=0$. We will analyze the dynamics in this limit by using the expansion of the action $\Gamma$ in powers of derivatives of the composite fields.

We begin by calculating the effective potential $V$. Since $V$ depends only on the $U(2)$ invariant $\rho^{2}=\sigma^{2}+\tau^{2}+\pi^{2}$, it is sufficient to consider a configuration with $\tau=\pi=0$ and $\sigma$ independent of $x$. Then, using the proper-time method [4], we find the potential from 
Eqs. (4), (10), and (11):

$$
V(\sigma)=\frac{\sigma^{2}}{2 G}+\tilde{V}(\sigma)=\frac{\sigma^{2}}{2 G}+\frac{N_{c}}{4 \pi^{3 / 2}} \int_{1 / \Lambda^{2}}^{\infty} \frac{d s}{s^{5 / 2}} e^{-s \sigma^{2}} e B s \operatorname{coth}(e B s) .
$$

The gap equation, $\frac{d V}{d \sigma}=0$, is

$$
2 \Lambda l\left(\frac{1}{g}-\frac{1}{g_{c}}\right) \sigma=\frac{\sigma}{|\sigma| l}+\sqrt{2} \sigma \zeta\left(\frac{1}{2}, 1+\frac{(\sigma l)^{2}}{2}\right)+O(1 / \Lambda)
$$

where the dimensionless coupling constant $g \equiv N_{c} \frac{\Lambda}{\pi} G, g_{c}=\sqrt{\pi}, l \equiv 1 /|e B|^{1 / 2}$ is the magnetic length and $\zeta$ is the generalized Riemann zeta function [7]. As $B \rightarrow 0(l \rightarrow \infty)$, we recover the known gap equation [8]

$$
\sigma|\sigma|=\sigma \Lambda\left(\frac{1}{g_{c}}-\frac{1}{g}\right)
$$

which admits a nontrivial solution only if $g$ is supercritical, $g>g_{c}=\sqrt{\pi}$ (as Eq. (9) implies, a solution to Eq. (13), $\sigma=\bar{\sigma}$, coincides with the fermion dynamical mass, $\bar{\sigma}=m_{d y n}$, and the dispersion relation for fermions is Eq.(可) with $m$ replaced by $\bar{\sigma}$ ). We will show that a magnetic field changes the situation dramatically: At $B \neq 0$, a nontrivial solution exists at all $g>0$.

We shall first consider the case of subcritical $g, g<g_{c}$, which in turn can be divided into two subcases: a) $g \ll g_{c}$ and $b$ ) $g \rightarrow g_{c}-0$ (nearcritical $g$ ). Assuming that $|\bar{\sigma} l| \ll 1$ at $g \ll g_{c}$, we find from Eq. (13):

$$
m_{d y n} \equiv \bar{\sigma} \simeq \frac{|e B| g \sqrt{\pi}}{2 \Lambda\left(g_{c}-g\right)}
$$

Since this equation implies that the condition $|\bar{\sigma} l| \ll 1$ fulfills at all $g$ satisfying $\left(g_{c}-g\right) \gg$ $\frac{|e B|^{1 / 2}}{\Lambda}$, the relation (15) is actually valid in that whole region.

At $g_{c}-g \lesssim \frac{|e B|^{1 / 2}}{\Lambda}$, introducing the scale $m^{*} \equiv 2 \Lambda\left(\frac{1}{g}-\frac{1}{g_{c}}\right)$, we find the equation

$$
m^{*} l=\frac{1}{|\sigma| l}+\sqrt{2} \zeta\left(\frac{1}{2}, \frac{(\sigma l)^{2}}{2}+1\right)
$$

which implies that in the nearcritical region $m_{d y n}$ is

$$
m_{d y n}=\bar{\sigma} \sim|e B|^{1 / 2}
$$


Thus in the scaling region, with $g_{c}-g \lesssim|e B|^{1 / 2} / \Lambda$, the cutoff disappears from the observable $m_{d y n}$. This agrees with the well-known fact that the critical value $g_{c}=\sqrt{\pi}$ is an ultraviolet stable fixed point at leading order in $1 / N_{c}[8]$. The relation (17) can be considered as a scaling law in the scaling region.

At $g>g_{c}$, the analytic expression for $m_{d y n}$ can be obtained at weak $|e B|$, satisfying the condition $\frac{|e B|^{1 / 2}}{m_{d y n}^{(0)}} \ll 1$, where $m_{d y n}^{(0)}$ is the solution of the gap equation (14) with $B=0$. Then, using the asymptotic formula $\zeta(z, q) \longrightarrow \frac{1}{(z-1) q^{z-1}}\left[1+\frac{z-1}{2 q}+\ldots\right]$ as $q \rightarrow \infty[7]$, we find

$$
m_{d y n}=\bar{\sigma}=m_{d y n}^{(0)}\left[1+\frac{(e B)^{2}}{12\left(m_{d y n}^{(0)}\right)^{4}}\right]
$$

i.e., $m_{d y n}$ increases with $B[9]$.

Let us now turn to calculating the kinetic term $\mathcal{L}_{k}$ in the action. The $U(2)$ symmetry implies that the general form of $\mathcal{L}_{k}$ is

$$
\mathcal{L}_{k}=N_{c} \frac{F_{1}^{\mu \nu}}{2}\left(\partial_{\mu} \rho_{j} \partial_{\nu} \rho_{j}\right)+N_{c} \frac{F_{2}^{\mu \nu}}{\rho^{2}}\left(\rho_{j} \partial_{\mu} \rho_{j}\right)\left(\rho_{i} \partial_{\nu} \rho_{i}\right),
$$

where $\boldsymbol{\rho}=(\sigma, \tau, \pi)$ and $F_{1}^{\mu \nu}, F_{2}^{\mu \nu}$ are functions of $\rho^{2}=\sigma^{2}+\tau^{2}+\pi^{2}$. To find the functions $F_{1}^{\mu \nu}, F_{2}^{\mu \nu}$, one can use different methods. We used the method of Ref. [10]. The result is: $F_{1}^{\mu \nu}=g^{\mu \nu} F_{1}^{\mu \mu}, F_{2}^{\mu \nu}=g^{\mu \nu} F_{2}^{\mu \mu}$ with

$$
\begin{aligned}
F_{1}^{00} & =\frac{l}{8 \pi}\left(\frac{1}{\sqrt{2}} \zeta\left(\frac{3}{2}, \frac{(\rho l)^{2}}{2}+1\right)+(\rho l)^{-3}\right), \\
F_{1}^{11} & =F_{1}^{22}=\frac{1}{4 \pi \rho}, \\
F_{2}^{00} & =-\frac{l}{16 \pi}\left(\frac{(\rho l)^{2}}{2 \sqrt{2}} \zeta\left(\frac{5}{2}, \frac{(\rho l)^{2}}{2}+1\right)+(\rho l)^{-3}\right), \\
F_{2}^{11} & =F_{2}^{22}=\frac{l}{8 \pi}\left[\frac{(\rho l)^{4}}{\sqrt{2}} \zeta\left(\frac{3}{2}, \frac{(\rho l)^{2}}{2}+1\right)+\sqrt{2}(\rho l)^{2} \zeta\left(\frac{1}{2}, \frac{(\rho l)^{2}}{2}+1\right)\right. \\
& \left.+2 \rho l-(\rho l)^{-1}\right] .
\end{aligned}
$$

By using the asymptotic formulae for zeta functions [7], we find the following spectrum for the excitations $\sigma, \tau$, and $\pi$ from Eqs. (12), (19) and (20): 
a) Subcritical, $g<g_{c}$, region:

At $g_{c}-g \gg \frac{|e B|^{1 / 2}}{\Lambda}$ (where $|\bar{\sigma} l| \ll 1$, see Eq. (15)) we find:

$$
\begin{aligned}
E_{\tau, \pi} & \simeq \sqrt{2}(\bar{\sigma} l)\left(\mathbf{k}^{2}\right)^{1 / 2}=\frac{g|e B|^{1 / 2}}{\sqrt{2} \Lambda\left(1-\frac{g}{g_{c}}\right)}\left(\mathbf{k}^{2}\right)^{1 / 2}, \\
M_{\sigma}^{2} & \simeq \frac{8 \sqrt{2}\left(1-\frac{g}{g_{c}}\right)}{\zeta\left(\frac{3}{2}\right) g} \Lambda|e B|^{1 / 2}
\end{aligned}
$$

(see Eq. (15)). Thus $\tau$ and $\pi$ are gapless NG modes. As the interaction is switched off, $g \rightarrow 0$, their energy becomes identically zero, and we return to the dynamics with spontaneous flavor symmetry breaking but without genuine NG modes. Also, as $g \rightarrow 0$, the $\sigma$-mode decouples $\left(M_{\sigma} \rightarrow \infty\right)$.

In the nearcritical region, with $g_{c}-g \lesssim \frac{|e B|^{1 / 2}}{\Lambda}$, we find from Eqs. (19), (20):

$$
E_{\tau, \pi}=f(\bar{\sigma} l)\left(\mathbf{k}^{2}\right)^{1 / 2}
$$

where

$$
f(\bar{\sigma} l)=\left(\frac{2}{\bar{\sigma} l}\right)^{1 / 2}\left(\frac{1}{\sqrt{2}} \zeta\left(\frac{3}{2}, \frac{(\bar{\sigma} l)^{2}}{2}+1\right)+(\bar{\sigma} l)^{-3}\right)^{-1 / 2} .
$$

Since in the nearcritical (scaling) region the parameter $\bar{\sigma}$ is $\bar{\sigma} \sim|e B|^{1 / 2}=l^{-1}$, we see that the cutoff $\Lambda$ disappears from the observables $E_{\tau}$ and $E_{\pi}$ in the scaling region.

b) Supercritical $g, g>g_{c}$, and weak $B(l \rightarrow \infty)$ :

$$
\begin{aligned}
E_{\tau, \pi} & =\left(1-\frac{1}{8(\bar{\sigma} l)^{4}}\right)\left(\mathbf{k}^{2}\right)^{1 / 2} \\
M_{\sigma}^{2} & =6 \bar{\sigma}^{2}\left(1-\frac{3}{4} \frac{1}{(\bar{\sigma} l)^{2}}\right)
\end{aligned}
$$

where $\bar{\sigma}$ is given in Eq. (18). These relations show that the magnetic field leads to decreasing both the velocity of the NG modes (it becomes less than 1) and the mass (energy gap) of the $\sigma$ mode.

As is known, in this model, an interacting continuum $(\Lambda \rightarrow \infty)$ theory appears only at the critical value $g=g_{c}$ (the continuum theory is trivial at $g<g_{c}$ ) [8]. At $B=0$, the continuum theory is in the symmetric phase at $g \rightarrow g_{c}-0$ and in the broken phase at 
$g \rightarrow g_{c}+0$. On the other hand, as follows from our analysis, in a magnetic field, it is in the broken phase both at $g \rightarrow g_{c}-0$ and $g \rightarrow g_{c}+0$ (though the dispersion relations for fermions and collective excitations $\boldsymbol{\rho}$ are different at $g \rightarrow g_{c}-0$ and $\left.g \rightarrow g_{c}+0\right)$.

The present model illustrates the general phenomenon in $2+1$ dimensions: In the infrared region, an external magnetic field reduces the dynamics of fermion pairing to one-dimensional dynamics (at the lowest Landau level) thus catalysing the generation of a dynamical mass for fermions. A concrete sample of dynamical symmetry breaking is of course different in different models.

We also studied the thermodynamic properties of the NJL model (8) at finite temperature $T$ and finite fermion density $n$. In leading order in $1 / N_{c}$, we found that the effective (thermodynamic) potential is:

$$
\begin{aligned}
V_{\beta, \mu}(\sigma) & =\frac{\sigma^{2}}{2 G}+\frac{N_{c}}{4 \pi^{3 / 2} l^{3}} \int_{0}^{\infty} \frac{d t}{t^{3 / 2}} e^{-\left(t l^{2} \sigma^{2}\right)} \operatorname{coth} t \\
& \cdot \Theta_{4}\left(\frac{i}{2} \mu \beta \mid \frac{i}{4 \pi t}\left(\frac{\beta}{l}\right)^{2}\right),
\end{aligned}
$$

where $\beta=1 / T, \mu$ is a chemical potential and $\Theta_{4}$ is the fourth Jacobian theta function [7]. The analysis of Eq. (26) (which will be described elsewhere) shows that there is a symmetry restoring (second order) phase transition both at high temperature and high density. The critical temperature $T_{c}$ is $T_{c} \sim m_{d y n}$, and the critical density $n_{c}$ is $n_{c}=\frac{|e B|}{2 \pi}$ at $g \leq g_{c}=\sqrt{\pi}$ (corresponding to the filling of the lowest fermion Landau level) and $n_{c}>\frac{|e B|}{2 \pi}$ at $g>g_{c}$. We recall that there cannot be spontaneous breakdown of a continuous symmetry at finite $(T>0)$ temperature in $2+1$ dimensions (the Mermin-Wagner-Coleman (MWC) theorem $[11,12])$. In the NJL model, the MWC theorem manifests itself only beyond the leading order in $1 / N_{c}$. A plausible hypothesis, we believe, is that beyond this order, the phase transition at $T=T_{c} \sim m_{d y n}$ will become the Berezinsky-Kosterlitz-Thouless (BKT) type phase transition [13], and, in particular, at $0<T<T_{c}$, the NG modes $\tau$ and $\pi$ will transform into BKT collective modes.

In this Letter, we considered the dynamics in the presence of a constant magnetic field 
only. It would be interesting to extend this analysis to the case of inhomogeneous electromagnetic fields in $2+1$ dimensions. As we have been informed recently, the program of the derivation of a low energy effective action in $2+1$ dimensional QED in external electromagnetic fields is now being developed by Cangemi, D'Hoker, and Dunne [14].

V.A.M. is grateful to the members of the Department of Applied Mathematics of the University of Western Ontario, where part of this work was done, and of the Institute for Theoretical Physics of the University of California (Santa Barbara) for their hospitality. He thanks J.M. Cornwall, E. D’Hoker, D. Kaplan, J. Polchinski, S. Raby, L. Randall, J. Schwarz, A. Vainshtein, K. Yamawaki, and A. Zee for useful discussions.

The research was supported in part by the National Science Foundation under Grant No. PHY89-04035. I.A.S. is grateful to the Soros Science Foundation for financial support.

\section{References}

[1] R. Jackiw, Phys. Rev. D29 (1984) 2375; I. Affleck, Nucl. Phys. B265 (1986) 409.

[2] A. Kovner and B. Rosenstein, Phys. Rev. B42 (1990) 4748; G.W. Semenoff and L.C.R. Wijewardhana, Phys. Rev. D45 (1992) 1342; N. Dorey and N.E. Mavromatos, Nucl. Phys. B368 (1992) 614; R. MacKenzie, P.K. Panigrahi and S. Sakhi, Phys. Rev. B48 (1993) 3892.

[3] T. Appelquist, M. Bowick, D. Karabali, and L.C.R. Wijewardhana, Phys. Rev. D33 (1986) 3704.

[4] J. Schwinger Phys. Rev. 82 (1951) 664.

[5] J. Bardeen, L.N. Cooper and J.R. Schrieffer Phys. Rev. 108 (1957) 1175.

[6] A.I. Akheizer and V.B. Berestetsky, Quantum Electrodynamics (Interscience, New York, 1965). 
[7] I.S. Gradshtein and I.M. Ryzhik, Table of Integrals, Series and Products (Academic Press, Orlando, 1980).

[8] B. Rosenstein, B.J. Warr and S.H Park, Phys. Rep. 205 (1991) 59.

[9] This fact was already pointed out in I.V. Krive and S.A. Naftulin, Phys. Rev. D46 (1992) 2737.

[10] V.P. Gusynin and V.A. Miransky, Mod. Phys. Lett. A6 (1991) 2443; Sov. Phys. JETP 74 (1992) 216; V.A. Miransky, Int. J. Mod. Phys. A8 (1993) 135.

[11] N.D. Mermin and H. Wagner, Phys. Rev. Lett. 17 (1966) 1133.

[12] S. Coleman, Commun. Math. Phys. 31 (1973) 259.

[13] V.L. Berezinsky, Sov. Phys. JETP 32 (1970) 493; J.M. Kosterlitz and D.J. Thouless, J. Phys. C6 (1973) 1181.

[14] D.Cangemi, E. D'Hoker and G.Dunne, Preprint UCLA/94/TEP/35. 